\section{An Aidress}

$$
\text { os }
$$

\section{INAUGURAL SYMPTOMS.}

Dehive bed before the Derby Mrdical Society,

By B. G. A. MOYNIHAN, M.S., F.R.C.S., SURGEON TO THE LEEDS GENERAL INFTRMARY.

Gantremen, - In an address which I had the honour to deliver before the Ashton-under-Lyne Division of the British Medical Association a year ago, I ventured to call attention to what I termed the "Pathology of the Living," and I endeavoured to show in what manner our conception of various diseases, having their origin within the abdomen, had become modified by a study of the morbid conditions disclosed in the course of an operation. I pointed out that much of our knowledge of the pathological processes involving the abdominal organs was based, chiefly, if not solely, upon investigations made in the post-mortem room, and I ventured to assert that the evidence therein obtained was given a value greater than its worth, when it came to be reckoned as a factor influencing or deciding our views upon the question of therapeutic messures. At the time when the dead alone were available for purposes of accurate pathological diagnosis it was the symptoms which were manifested during the last fow weeks or months of the patient's life which chiefly attracted attention. These symptoms were looked upon as the natural and necessary subjective expressions of those morbid conditions the final stages of which were presently displayed at the autopsy. And so when textbooks came to be written, it was the late symptoms and signs which, having attracted special and recent attention, became conspicuously recorded. It was the laie manifestations which were thought to be the characteristic manifestations of any form of disease, and it was upon them that attention chiefly centred. But late symptoms are, I submit, no more characteristic of any disorder than early ones, and their importance as signals for therapeutic aid is relatively insignificant, for they come at a time when heroic measures have not seldom to be adopted if the life of the patient is to be saved or prolonged. Late symptoms are too often the heralds of death; inaugural symptoms may be the cry for timely surgical assistance.

In this paper I wish to draw attention to the urgent need which exists for a study of the very early symptoms of all diseases, but more especially of those affecting many of the abdominal organs. The surgeon, when he is about to operate upon a patient suffering from any abdominal disease, has the opportunity to observe not only those parts for which his operative interference is immediately necessary, but also all other viscera which can be laid bare through the same incision. If a morbid process in its earliest stage be then discovered, perhaps in parts other than those primarily concerned in the operation, the patient's story of his sufferings may be retold, and crossexamination conducted thereupon, as soon as recovery from the operation is complete. It rests, therefore, in no small degree, with the surgeon to elicit the inaugural symptoms associated with the pathological changes which he finds within the abdomen, and by making sure of their significance and of the due order of their appearance, to furnish the knowledge that will ensure clinical recognition of visceral diseases in their early stages, in the stages when they are surely amenable to curative treatment.

There is immediate need for this earnest investigation of inaugural symptoms. It is a frequent and depressing experience for the surgeon to have referred to him cases, of malignant disease more especially, though by no means exclusively, when the time for safe operative treatment, with the almost certain prospect of complete relief, has passed long ago. Only a few days ago, by the kindness of one of my collergues, I became possessed of a specimen of chronic duodenal ulcer which had caused a patient's death. The man had been admitted to hospital suffering from profuse haomatemesis and melaena; he was desperately ill, moribund indeed, and he died before the bleed. ing could be checked. An ulcer, the size of a shilling and about 1 in. deep, eroded a large vessel the orifice of which was displayed in the base of the ulcer. That ulcer had existed for months, perhaps for years; it had long callod aloud for recognition, yet its ory, had never been beard or heeded, despite the fact that the sjmptoms of this disease are as definite and unmistakable as are those of a broken limb.

That cancer of the stomach is a common malady wo hnow well enough; it claims an appalling number of victims every year. It is a disease which is purely local in its early stage, a disease which accordingly lends itself readily enough to radical treatment. Yet it is probably safo to asy that there are not in all England ten petients who have been cured of this dire complaint. The tale of the victims of appendicitis is told almost daily in the newspapers. If the early symptoms of this disease were commonly nnderstood and appropriate treatment adopted from the first (not necessarily operative treatment), the terrible mortality would be very considerably reduced. I think it is almost certain that the acute fulminating cases (so-called) of this disease give always a definite warning of their approach ; it is our ignorance of this warning that proves so disastrous. How does it come abont; then, that we are so pitifully helpless in these and in many other like diseases? It is, I venture to say confidently, because we rely for our diagnosis not upon inaugural symptoms, but npon those of late appearance; we confuse far too frequently the symptoms of a tardy complication with those of the original morbid process itself. We hesitate to diagnose cancer of the stomach before a lump can be felt, and we have not the courage, in a case of reasonable donbt, to open the abdomen to look. We question the evidence of duodenal ulcer until haemorrhage occurs, though haemorrhage is a late, dangerous, and preventable manifestation. We dare not hint the presence of gall stones till jaundice comes, though symptoms of the plainest meaning have been present for years and in spite of the fact that jaundice is an infrequent symptom of gall-stone disease. Indeed, much of the textbook symptomatology urgently demands revision. It is based upon diagnoses made in the advanced or terminal stages and verified upon the dead. Our knowledge now of the "pathology of the living" must urge us to scrutinize the early history more closely and to endeavour to correlate the inaugaral disturbances of health with the morbid conditions responsible therefor, which are laid bere by operation.

I think there is a fault of which we are all in greater or less measure guilty-we are very apt to ignore or belittle the history of the case from the patient's point of view, the anamnesis that is. The word "anamnesis" is one the significance and the usefulness of which seem to be insuffi. ciently appreciated. It means the calling again to mind incidents in the past, the recollection of occurrences almost or entirely forgotten until thought was concentrated thereon. Its meaning in medicine accordingly should be the reproduction in the patient's mind of the details of the earliest clinical history. The "previous history" 89 it is generally told in published case reports is a jumble of the statements of the patient and of the prejudices, opinions, and reflections of the recorder. It is time that the word "anamnesis" came into general adoption, and that it should be held strictly to indicate the recollection by the patient of the details of his illness-that, neither more nor less. The anamnesis cannot be too detailed, for it affords the only authentic information which can be obtained, and when it is reviewed in the light of the fuller knowledge which has come to the surgeon after the exposure and careful, purposeful scrutiny of the parts involved we should little by little become confident in making our diagnoses at a much earlier period than now seems customary or possible. A plan which I frequently follow is to ask the patient to write for me in the most detailed manner the story of his own sufferings from the time of their very earliest onset, exaggerating nothing, omitting nothing because of its irrelevance or apparent triviality. Many little points may be brought out in this way, points which are apt to escrpe one's notice when the examinations are being made.

It is in dealing with the acute catastrophes occurring within the abdomen that we shall probably derive the most instant and striking advantage from an attontive study of inaugural symptoms. It is in these cases that minutes gained mean lives saved; for the earlier the gravity of the case is realized and surgical treatment

[2500] 
adopted the safer will the issue be. Other things being equal, the mortalitiv rises in direct proportion to the time which has passed since the disaster occurred. Many of the symptoms and signs formerly described as attendant upon the perforation of a hollow viscns are not manifesta. tions of that particular incident at all, but are evidences of a later and preventable complication, acute diffuse peritonitis. To take a specific example, the perforation of a gastric or duodenal ulcer. But let me first say that a catastrophe of this kind is almost always capable of being forestalled. Though the onset of perforation in an ulcer is acute, the ulcer itself is of the chronic type. It is an ulcer that has existed for months or years, and it has given, in almost every instance, not only sustained evidence of its existence, but a recent warning that the pathological processes engaged in it were becoming more acute. The warning, however, is commonly ignored, because the significance and importance of it are not understood, and accordingly a disaster is precipitated. There are few catastrophes occurring within the abdomen that are veritably "acute." When we speak of such things we refer, as a rule, to the abrupt incursion of acute symptoms into the even and placid course of a disorder whose more tranquil manifestations have been present for months or it may even be for years.

If the account of the perforation of an ulcer given in most of the textbooks be examined it will be found that collapse, rapidity, and poor quality of the pulse, and distension of the abdomen are conspicuously mentioned as symptoms. Yet when a case is seen in the early hours not one of these is necessarily present. Collapse is certainly not present, if it is to be measured by the customary signs, for there is usually a pulse that is not above 80 , and of a quality that is not very perceptibly altered from the normal. But if there is intense pain, with the most unyielding stiffness of the abdominal muscles, then a perforation has probably occurred. When it is remembered that the diaphragm is also an abdominal muscle, and that it is held with a rigidity that never slackens, the shallow thoracic respiration is explained at once. The patient cannot make any pretence to breathe deeply, and the replies to one's questions are jerked out with an effort, the end of which is cut short by a spasm of pain. This nnalterable resistance of the abdominal wall is doubtless a reflex having for its purpose the protection of the acutely affected area by a muscular splint. The alert, anxious, apprehensive look the patient always wears is the most eloquent evidence of his intense sufferings. These things, then-the sudden onset of an acute intolerable pain that does not abate, rigidity of all the abdominal muscles, light and shallow breathing, with an inspiratory phase that often ends abruptly in a "catch," together with the intensoly anxions expression which the face always wears -are ample warrant for a diagnosis of a perforation. A previous history of indigestion is rarely, if ever, lacking. A rapid pulse-rate, vomiting, abdominal distension are not to be looked for among the inaugural symptoms. They are the proof that precious time has already been wasted and a valuable opportunity thrown away.

Of the symptoms of duodenal uicer-a common disease, more frequent, indeed, in my recent and present experience than gastric ulcer-I have written fally elsewhere. ${ }^{2}$ It is a very curious feature in connexion with this disease that perfectly accurate accounts of its symptomatology are given by authors who do not seem to have the remotest conception that the condition they are describing is not, as they suppose, one of "functional" disorder, but one in which a demonstrable organic lesion is present. The vague terms "hyperchlorhydria," "acid dyspepsia," "nervous dyspepsia," are given very generally as diagnoses; they are too often words without meaning, clinical synonyms for the pathological condition duodenal ulcer. This is a disease which, left to itself or attacked in the most approved manner by diet or by drugs, is a very serious menace to health and frequently imperils the life of the patient. I have already referred to haemorrhage in connexion with this disorder. Now, haemorrhage is, almost without exception, a very late symptom of duodenal ulcer; it is an evidence that a diagnosis which should have been made earlier has been missed. Yet what do the textbooks say? In Nothnagel's Encyclopaedia (page 245) we are told that "severe haemorrhage occurs in about one-third of the cases," and Cullen, Fenwick, and
Perry and Shaw are quoted, who variously estimate the death-rate from haemorrhage as from 13 to 36 per cent. Surely it is almost as reasonable to wait for haemorrhage before venturing to diagnose a duodenal ulcer as to include a ruptured perineum among the signs of pregnancy.

I am disposed to think that we shall be repaid sevenfold by a very close attention to the inaugural symptoms of gall-stone disease. At the present time no slightest notice is paid to them in the ordinary textbooks on medicine. Here I deliberately select one of them for quotation, because it lies always near my hand and because it is written by the ablest authority I know-one of the choice and master spirits of this age, who has always displayed a great interest in surgical work and an intimate knowledge of surgical literature. Therein it is written: "In a majority of cases gall stones cause no symptoms. The gall bladder will tolerate the presence of large numbers for an indefinite period of time"; and again, "It will be better, perhaps, to consider cholelithiasis under the following headings. The symptoms produced by the passage of a stone through the ducts-biliary colic the effects of permanent plugging of the cystic duct; of the stone in the common duct, and the more remote effects due to ulceration, perforation, and the establishment of fistulae." Then follows a detailed description of the sym. ptoms of the disease in these various terminal stages. There is no mention, it will be seen, of any early sym. ptoms, nor of the symptoms which are due to any other than late and, as I hold, preventable, complications. The very accurate descriptions which follow refer, all of them, to the stages of gall-stone disease which it should be our business to forestall by removal of the offending cause in the early days.

The only authority, so far as I am aware, who has directed special attention to the early symptoms caused by gall stones is Kraus, ${ }^{3}$ who describes what he terms a "prodromal stage of cholelithiasis." He gives a clear account of the fairly early symptoms by which gall stones announce their presence ; his description is accurate, not as he supposes, for the period during which gall stones are possibly forming, but for the period when they are already present and are bent on making their whereabouts known. It is of the greatest importance to recognize that the in. augural symptoms due to gall stones are referred in the anamnesis, not to the liver or to the gall bladder, but to the stomach. The patients complain of a fullness, weight, distension, or oppression in the epigastrium coming soon after meals, usually within half or threequarters of an hour, relieved by belching, and dis. missed almost on the instant by vomiting, elicited with remarkable constancy by certain articles of diet and dependent rather upon the quality than upon the quantity of the food. There is a sensation of great tightness, which, if unrelieved, may become acute pain, from which the patient obtains ease by bending the body forwards, by flexing the right thigh on the abdomen, or by loosening all garments which fit tightly to the waist. While the discomfort lasts the patient may notice a "catch" in his breath, and he finds, perhaps, that it is impossible to breathe deeply without feeling an acute stabbing pain at the right costal margin. There may be a feeling of faintness and nausea, and, rarely, vomiting may occur spontaneously. After a more than usually severe attack of "indigestion" the body and side may feel stiff for several days. A frequent and a very characteristic early symptom of cholelithiasis is the occurrence during an attack of in. digestion of a slight sensation of chilliness, especially in the evenings, after a meal. The patient may shiver for several minutes, and may hasten from the table to huddle over a fire. The sensation of "goose flesh" is often experienced, and several medical men upon whom I have operated have said that in the severer phases it was not unlike a very slight rigor, the chilly stage being quickly followed by one in which the body feels hot, and the skin begins to act freely. My friend, Dr. Leonard Molloy, who is very quick to recognize the presence of gall stoncs, groups all these symptoms in the phrase "Gall-bladder dyspepsia."

It is no doubt owing to the fact that these inaugural symptoms of cholelithiasis are not generally recognized that the pernicious and inveterate heresy, which asserts that calculi may commonly exist in the gall bladder with. out causing trouble, is still abroad, and is still complacently 
aecoptied as an article of faith by every physician and by many surgeons. Nannyn writes (p. 56): "Cholelithiasis is, as post-mortem observations show, an extraordinarily common malady. On an average every tenth human being, and of elderly women perhaps every fourth, has gall stones. This does not, of course, express the frequency with which biliary calculi give rise to morbid phenomena, for. one often enough finds, post mortem, the gall bladder and bile ducts completely packed with calculi, although these have never caused any inconvenience or produced any ill effects. In such cases cholelithiasis may be a perfectly harmless condition, and not merely a latent one." It is to be admitted at once that gsil stones may exist without arousing those symptoms which Naunyn proceeds to describe in the most admirable manner, the symptoms which have a textbook authority, the late symptoms. But I have no hesitation in affirming that it is excessively rare for symptoms, which are plain enongh in their meaning if only we had the intelligence to recognize them, to be absent when stones lie within the gall bladder. Probably the only circumstance which in such cases prevents their appearance, or rather is responsible at last for their cessation, is the closure of the cystic duct. The gall bladder is then no longer a reservoir for bile; it is out of the circuit, so to speak, and the stones which lie within it are inert foreign bodies of the same kind as the bullet which lies embedded and encapsuled in the muscles of the thigh. This condition is one to which that very able surgeon, Mr. Rutherford Morison, applied the term "natural cure." A sort of cholecystectomy has been performed by Nature's rough hand in the todions and dangerous attempt to give relief by isolating a cause of persistent irritation. Symptoms of the kind described then cease to appear, but the fact of their presence at an earlier period hardly fails to be elicited when purposeful inquiry is made. With the rarest exceptions, therefore, I believe it to be true that stones do not develop in the gall bladder, and do not remain there after their formation without exciting symptoms of a kind which we have neglected adequately to study. In my own experience, which is not inconsiderable, I have never yet found gall stones in the gall bladder, when performing other abdominal operations, without being able subsequently to elicit the most positive evidence of their frequent endeavours to attract attention and to reveal their presence.

The most melancholy part of my own daily work is that concerned with the treatment of cases of carcinoma of the stomach. It is true that in some instances where I have performed partial gastrectomy, and in one where the whole stomach was removed, the results have been very gratifying. These cases show what may be done in the way of radical treatment for this frequent and horrible disease. Yet they leave one full of regret that they bear so very small a proportion to the total number of cases that are referred to us for advice or for treatment. Nothing to me is more depressing than to be com. pelled to confess in a case of cancer of the stomach that surgical treatment offers no prospect of any the slightest relief, even for a few weeks or months, to the patient's pitiful condition. Yet of cancer of the stomach it is perfectly accurate to say that it is often a disease which timely treatment could have prevented, and one with which surgery should be able successfully to grapple. The truth of the former of these statements depends upon the demonstration of the connexion between chronic ulcer of the stomach and carcinoma. The anamnesis of patients suffering from cancer of the stomach seems to indicate that in rather more than 60 per cent. of the cases a chronic ulcer has preceded the onset of malignant disease. The pathological evidence now available shows that cancer develops in connexion with an ulcer in something more than 50 per cent. We have waited long for the pathological proof of that which clinically we had long suspected; such proof can only be furnished by specimens examined in a stage where the transformation is not yet complete. The simple ulcer and the malignant degeneration must exist side by side, and the former must be of greater age than the latter. Specimens of this kind are hardly to be found on the post-mortem table, in the bodies of those who have fallen victims to the unchecked ravages of this disease; they must be obtained from the operation theatre. It is in the examination of specimens of partial gastrectomy that the convincing pathological evidence has now been 4 found. Dr. W. J. Mayo, who in this matter is not exceeded in experience or authority by any living surgeon, found that, in 180 cases of resection of the stomach, cancer was demonstrated to have sprung up in the base of an vilcer in 97, that is, in 54 per cent. 4 The surgical trestment of chronic gastric ulcer may, then, be confidently expected to diminish the number of cases of cancer of the stomach, if the latter is not seldom the tardy result of the former. The possibility of a radical cure of cancer of the stomach once developed depends upon our knowledge of its mode of spreading in the stomach itself, and of the distribution of the lymphatic vessels and glands of the parts involved. As a result of the researches of Cuneo and others and of the recent excellent work of my colleagues, Dr. Jamieson and Mr. Dobson, we are well equipped. with information on these points. Cancer of the stomach, then, should not be the almost certainly fatal disease which it is at this moment. All that is necessary is the recognition of the cases in an early stage, and this can only be achieved by earnest research into the inaugural symptoms. Of cancer of the stomach I described ${ }^{5}$ two types as they affect the distal half of this organ, the "pyloric" and the "prepyloric." In the former the symptoms are obstructive from the beginning, and romiting appears when the disease is in its earliest stage. Stasis then is present, and it is not long before some hypertrophy of the wall of the stomach occurs. These cases should admit of early recognition and of very successful treat. ment, for there is no other cure of a mechanical obstruction in the stomach than that which surgery offers. In the "prepyloric" form the inaugural symptoms are individually vague, bat collectively enable a certain diagnosis to be made. A man beyond middle life finds by degrees that he takes less interest in his meals; his food loses its relish and presently becomes distasteful. Life in many of its aspects seems to lose its zest; neither work nor leisure are enjoyed, and depression, increasing anaemia, and loss. of weight are soon observed. It is not for many weeks, or perhaps many months, that vomiting is noticed; it is then due to the gradual enlargement of a growth which, beginning on the lesser curvature of the stomach, spreads downwards, on one or both surfaces, until it attains such size that the pyloric antrum becomes narrowed, and obstruc. tion results. In 'several of my cases haemorrhage has been the first symptom. I had under my care recently a gentleman of 67, referred to me by Dr. Malim of Rochdale, who suddenly, without any warning, when feeling in robust health, vomited a very large quantity of blood and fainted. Soon afterwards the symptoms I have just detailed made their appearance, and at the operation a growth of the prepyloric form was discovered. An exactly similar beginning occurred in a case kindly sent to me by Dr. Daly of Hull a few months ago, and I have notes of several other instances. The sudden occurrence of profase haematemesis in a man previously in good health is to be looked upon as especially significant. We should have our suspicions keenly aroused if, in a patient at or beyond middle life, whose anamnesis tells of the existence at some earlier time of a chronic gastric ulcer, there develop distaste for food, loss of appetite, intolerance and positive refusal of solid food, uneasiness after meals, even the restricted ones taken unwillingly, loss of weight, persisting anaemia, and vomiting or haematemesis. Then explora. tion should be urged, for in the present state of our know. ledge of the early symptoms there is no other method by which an early carcinoma of the stomach can be discovered. No one deprecates more strongly than I the haphazard exploration of the abdomen for diagnostic purposes. I think the most sedulous care should be expended on the examination of the patient, and that every detail of the anamnesis should be scrutinized before the abdomen is opened, otherwise we may let slip great opportunities for clinical research. We cannot correlate the clinical discoveries on the one hand, and the disclosures of the operation on the other, if we are perfunctory or neglectful in our acquisition of the former. But it would be foolish not to accept the position that at this moment there is no means of making an assured diagnosis of cancer of the stomach in the curable stage by any other means than the inspection of the parts as to whose integrity there is reasonable doubt It may not be wholly inopportune to suggest that the system which at present obtains in many 
hospitals of admitting and of retaining cases of cancer of the stomsch under the care of a physician is wrong. There is no medical treatment of this condition. If the pationt is to have any chance of substantial relief, or the slender proopect of cure, it can only be through means which the surgeon alone is competent to employ.

The treatment of cancer of the large intestine is also greatly handicapped by reason of a lack of adequate knowledge of inaugural signs and symptoms. During the last few years I have performed colectomy in a large number of eases, and with one excoption operative intervention became necessary because of the onset of acute or chronic obstruction, or because a tumour had been discovered in the large intestine. The presence of a tumour so large as to be felt through the abdominal wall, and the occurrence of an acute obstruction are both evidence of ancient disease; for the intestinal growth is usually slow in its rate of inorease, though not invariably so, and an acute obstruction comes ordinarily only as a terminal manifestation in the course of chronic intestinal disorder, Now, it is of the most urgent importance that the very earliest disturbances of health due to cancer should be recognized, for cancer here, as elsewhere, is at first a local diserse; and because local and while local is surely amen. able to complete eradication. In the case of carcinoma of the colon there are many points which in this special instance should make for complete success when the neoplasm is removed. The growth is as a rule slow, the lymphatic supply of the colon as a whole is not very free, the lymphatic system in all parts lends itself to very thorough removal, and is not invaded very early in the course of the disease. Furthermore, by means of a procedure to which I recently drew attention, ${ }^{6}$ great lengths of the large bowel can be removed without risk of damage to the vascular supply, and with the certainty that end-to-end ansstomosis can be obtained. An early operstion, if conducted upon the proper lines, should therefore give very gratifying results. What, then, are the earliest evidences of the invasion of the colon by carcinoma? The first, I think, is the insidions onset of intestinal irregularity. The bowel acts with a certain caprice, there is now slight constipation, and now slight diarrhoea. These symptoms become, of course, considerably emphasized at a later stage, where there may be intestinal obstruction of three or four days' duration, alternating with a copious and teasing diarrhoea. But something much less than this occurs quite early in the disease, and at the same time there is commonly present a symptom which I hold to be of great significance. It is the occurrence of a spasm, slight and transient, in a part of the large intestine. The pationt tells us that every now and then there is a feeling of "gripping" (or he may himself use the word "spasm") in a certain very limited area of the abdomen, and he points always to the same spot. This is clearly to be explained by the existence of a slight hypertrophy of the intestinal muscle as a result of the increased effort necessary to pass the contents of the gut through a segment in which stenosis is already beginning to appear. A patient upon whom I recently operated for a growth in the sigmoid flexuse described this sensation to me in these words: "I feel as if a hand within my body tightened every few minutes, trying to grip my watch." At the same time borborygmi are heard, and their onset is found to synchronize with the sensation of spasmodic pain.

The occurrence of mucus or of occult blood in the stools is probably to be expected in the comparatively early stages also. I have recently received help in the diagnosis of cases of obstruction in the large intestine from the examination by the $x$ rays of patients to whom bismuth had been administered by the mouth. The presence of an impediment to the easy passage onward of the faecal current may be graphically depicted by the accumulation of the shadow-throwing bismuth behind the point of constriction in the intestine. When, therefore, any or all of these several points are elicited in a patient of middle age or over, and when loss of weight, indifference to food, or positive repugnance to it, or the deliberate avoidance of the more substantial meats, are together observed, there is every probability that serions organic disease is present in the large intestine.

Of all the diseases affecting the abdominal organs there is none that may appear in such varied form as appendicitis. A slight attack may pass often unrecognized, its nature being elucidated only when a similar onset leado on a later occasion to a more severe attack, and an opera. tion for the evacuation of pus ar for the remoral of the sppendix then becomes necessary. In all instances, how. ever, pain is the inaugural symptom; if other symptoms appear first the diagnosis must be looked upon with suspicion. Only recently I have verified once again this rule, upon which that most acute observer, Dr. J. B. Murphy, has so long insisted.

A lady, aged 50, whom I saw with Mr. Jalland of York, woke on a certain Friday morning at 5 o'clock in a rigor. She was shivering violently, and her temperature ran up to $103^{\circ}$. She had no pain then, or at any time during the day, but remoined in bed, as she felt weak and ill. At almost the again another saturday morning she had another rigor, and rigor was in progress she had a sudden and very severe attack of pain in the abdomen, and a very sharp attack of diarrhoea followed. There was no vomiting, but a decided feeling of nausea. Daring Surday the pain continued, being worse every hourea. During Suyday the pain continued, being worse every gradually distended. I saw her early on Monday. The abdomen was full, especially in its lower half, and the muscles were tightly held. The whole of the lower part of the abdomen was tender; there was tenderness on vaginal examination, and the vterus, as was previously known, was enlarged to the size of an orange by a flbroid. 'The temperature was $100^{\circ}$, the palse 100. The bowels had acted well after an enems. Mr. Jalland was convinced that the condition was steadily getting worse, and the evidences of a spreading peritonitis were unmisworse, and the evidences of a spreading peritonitis were unmis-
takable. The most likely diagnosis seemed to me to be perforative peritonitis; but pain was not the inaugural symptom. In discussing the case before operation I made the remark to $\mathrm{Mr}$ Jalland that the signs were those of an acute perforative appendicitis, but that the initial symptom, pain, invariable in appendicitis in my experience, was absent. I opened the abdo. men and verified Mr. Jalland's original diagnosis, a remarkably astute one, I think. There was a perforation at the fundus of the uterus from which offensive pus was oozing. An abscess in the wall of the uterus ? had ruptured into the general peritonea? cavity, wherein we found a very large quantity of the most offensive pus. I performed hysterectomy and drained the abdomen. The patient has happily made an excellent recovery.

The significance of the inaugural symptom, the thricerepeated rigor, was at once appreciated when the pathological condition was exposed, for the initial rigors were clearly due to an acute inflammatory process in the walls. of the aterus, while the later perforation into the general peritoneum was announed by the sudden onset of the very severe abdominal pain.

Time permits me to refer only in the briefest manner to the pancreas. This is the most important gland in the haman economy; of its diseases we know little, and of their inaugural symptoms nothing at all. Yet it is hardly possible that a gland whose external secretion is the most potent of all digestive juices, and whose internal secretion is deeply concerned with the regulation of the metabolic processes of the body, should be able to deviate widely from the normal without betraying some clear sign. Many of the disorders of the pancreas, as Riedel' taught us long ago, are associated with cholelithiasis. Mayo Robson has recently put forward good reasons for supposing that "catarrhal jaundice" may be due to an inflammation of the pancreas, whose consequent enlarge. ment canses pressure upon and obstruction within the duct, which it transmits to the duodenum. I think the better view may be that both gall stones and pancreatitis are themselves the result of an infection of the bile as it descends from the liver. The scavenging properties of the bile have been demonstrated by Lartigau and others. This excretion bears away certain organisms brought to the liver in the portal stream, organisms which render it an infective agent, which dispose to the formation of stones and which make possible the infliction of an injury to the pancreas. Of the significance and of the consequence of inflammation so set going we are only now beginning to realize the grave importance. In two cases in my own practice I have known of deaths from diabetes three years and six years after operation at which chronic pancreatitis was discovered. We now know, chiefly from the work of Opie, that in a certain form of pancreatitis, the interacinar, the islands of Langerhans are attacked, their internal secretion which controls carbohydrate metabolism is suppressed, and diabetes results. But we have very little knowledge of the frequency with which diabetes depends upon a lesion of the pancreas, nor do we know how often such a lesion can be traced to an attack of "catarrhal jaundice" passed through in the days of 
infancy or childhood. Happily the labours of Cammidge have now made it possible for us to recognize, by examination of the urine and faeces, whether a morbid condition of the pancreas is present in any given case. We know that in mumps the gland is not seldom attacked, its implication in typhoid fever, as I have shown, ${ }^{8}$ is capable of demonstration, and it seems not improbable that it is in some degree affected in many of the acute specific fevers. What relation, if any, such ineidents bear to the later onset of diabetes we have no cemotest idea; but the subject may well repay attentive inquiry. The inaugural disturbances in so important a gland as the pancreas may perhaps have consequences the vastness and the importance of which are hardly realized to-day.

These are some of the instances, not by any means all, perhaps not even the happiest, which might have been selected, in which a commencement has been made in the investigation of the inaugural symptoms of abdominal diseases. It has seemed to me desirable and appropriate to call attention to the necessity for a closer and more intimate study of all diseases in their earliest beginnings. We must shake off the incurious apathy which seems now to possess us in reference to all those early, no doubt often "trivial," disturbances of health which lead slowly or swiftly, none the less surely, to disorders of so grave a character that serious or formidable measures become necessary for their arrest. It is chiefly, I think, to the general practitioner that we must look for the most effective help in this research. It is he who sees the cases first, and it is he to whom the occasion and the opportunity come for the early diagnosis. I believe it to be true that our textbook descriptions of the clinical aspects of many diseases are based upon the manifestations which are present when that disease is in its full career or is hastening to the end. The terminal rather than the prodromal symptoms receive detailed description. This was, of course, inevitable in the days when the confirmation of the diagnosis could only be made at the time the dead body was examined. Now our opportunity is greater and our responsibility clearer. It is, I think, by a closer and more intimate examination of the anamnesis, followed by a precise investigation of the parts affected during the life of the patient, that our knowledge of the earlier phases of diseases will come. By this means we shall be able by degrees to remove the reproach that now justly attaches to much of our work in the surgery of the abdominal viscera-the reproach that we are so often "too late."

REFERENCES.

1 British Medical JoCRnal, 1907, ii, p. 1381. 2 Practitioner, July, 1907. ${ }^{3}$ Prag. med. Woch. 1884, 49. 4 Annals of Surgery, 1908, xlvii, og. and Obstetrics, 1908 OCRNA, $1906,1, p .30$. S Surgery, GynaecoAmer. Med. Assoc., 1908, ii, p.127. 8 Lancet, 1903, i, p. 1586

\section{$\mathfrak{R}_{\mathfrak{m} \text { markm }}$}

\section{ON THE

RHELMATIC ORIGIN OF CERTAIN
SEROUS INFLAMMATIONS.

By EUSTACE SMITH, M.D., F.R.C.P.,

SENIOR PHYSICIAN TO THE EAST LONDON HOSPITAL FOR CHILDREN.

THE rheumatic origin of serous inflammation of the pericardium is recognized by every one and has never, I think, been called in question, but a similar source for inflammation of other serous membranes is less willingly conceded. Why this should be so-why, for instance, a serous membrane in the abdominal cavity should be held to be exempt from a source of illness to which a serous membrane in the thorax is admitted to be prone, is a mystery of which I have never heard a satisfactory explanation. One may assert, or one may deny, this immunity from rheumatic inflammation of the abdominal serous membrane, and a proof in either direction is not easy to arrive at. The success or failure of antirheumatic treatment should, however, afford testimony the significance of which could not be entirely ignored; and if in any case there arose some fresh manifestation common to rheumatic illnesses, the suggestive evidence thus supplied would deserve to be carefully weighed before being rejected as empty or inconclusive.

Some years ago I saw in consultation with Dr. A. B. Rendel a robust little boy of 21 years who. was suffering from acute robust ictis. It was the third day of the illness, and I found appendicitis. It was the third day of the illness, and I foumd the child lying with his right knee drawn up and presenting all the characters of a sharp attack of the inflammation. There
was, in addition, a soft and evidently quite recent systolic was, in addition, a soft and evidently quite recent systolie murmur heard at the apex of the heart. Now several months
previously I had had an opportunity of making a thorough examination of the boy, and am sure that at that time the hear sounds were absolutely healthy. Moreover, Dr. Rendel had examined the heart with the other organs at the beginning of the illness two days before my visit and was satisfied that the heart sounds then had been perfectly clear. There was no pain or tenderness complained of in the joints, and the illness had in all respects the features of an ordinary attack of inflammation of the appendix. Sodium salicylate was ordered, and the inflammation very quickly subsided; but the heart murmur persisted, and the child's temperature ranged from $99^{\circ}$ to $100^{\circ}$ persisted, and the child's temperature ranged from $99^{\circ}$ to $100^{\circ}$
for ten days after the signs of appendicitis had disappeared. I heard recently from Dr. Rendel that the murmur was still udible, after three years, but that compensation was complete.

In this interesting case the cardiac murmur occurred suddenly in the course of an inflammatory disease, and the presumption is strong that it was also due to inflammatory agency. The patient had been quite healthy up to the time of the attack. He was not anaemic or cachectic, or in any way ill-nourished or in such a condition as originates and maintains a temporary disturbance of function. Moreover, the murmur did not subside as the disease passed off, but remained as a permanent feature, which even in a cursory examination could hardly escape attention. It is difficult, then, to reject the belief that there must have been some connexion between the inflammation in the abdominal cavity and the valvular inflammation, or that the two lesions were a consequence of the same general cause. That this cause was not an infection of the valve by any virulent microbe such as the Bacillus coli is evident from the mild character of the symptoms. Infective endocarditis is a complaint of the utmost gravity which gives rise to urgent and distressing symptoms, and too often proves fatal. In any case the illness is long and tedious, and convalescence imperfect and slow. In the case recorded the cardiac complication in no way increased the severity of the attack, for this ran a short and easy course, and soon came to an end. The subsidence of the disease followed very quickly upon the adoption of antirheumatic treatment; but, leaving that out of the question, it is difficult to suggest any other explanation of an inflammation attacking at the same time a serous membrane in two different parts of the body than to presume that in each case its origin was the same; and, as the valvular inflammation had all the familiar characters of a mild rheumatic endocarditis, to attribute the inflammation of the appendix to a similar rheumatic source. Were it otherwise, we should be forced to conclude that a mild valvular inflammation can be set up by other than rheumatic agency in a child who is free from infectious fever or constitutional disease, and whose insignificant general symptoms forbid the suspicion of infection of the valve by a virulent micro-organism. Personally, I believe rheumatic appendicitis to be a common complaint. The inflammation is severe at first, and gives rise to all the symptoms which we are used to associate with this form of illness; and, in the absence of other manifestations of the rheumatic dyscrasia, I know of no other way of distinguishing it from the infective variety than by observing the almost immediate improvement which sets in under the influence of the special treatment. In former days, when no one ever thought of operating - at any rate, at an early stage-and every case of "typhlitis" was treated by rest and opium, the patients usually recovered after an illness of a week or ten days. Now, under the sodium salicylate, improvement in this, the rheumatic, form of the complaint, generally begins well within forty-eight hours of taking the first dose of the medicine, and is usually completed before the end of the week.

Instead of a circumscribed local inflammation, the illness may take the shape of a severe general peritonitis. The following case seems to me to be capable of no other explanation than that it was an instance of rheumatic inflammation affecting the peritoneum.

A young man about 22 or 23 , well built and robust, left Liverpool on a very cold day at the end of his holiday to return home. 